\title{
Application of Blood Group Genotyping by Next-Generation Sequencing in Various Immunohaematology Cases
}

\author{
Tae Yeul Kim ${ }^{a} \quad$ HongBi Yu $^{b} \quad$ Minh-Trang Thi Phan ${ }^{c} \quad$ Ja-Hyun Jang ${ }^{a}$ \\ Duck Cho ${ }^{a, b, c, d}$
}

aDepartment of Laboratory Medicine and Genetics, Samsung Medical Center, Sungkyunkwan University School of Medicine, Seoul, South Korea; bepartment of Health Sciences and Technology, Samsung Advanced Institute for Health Sciences and Technology, Sungkyunkwan University, Seoul, South Korea; 'Stem Cell and Regenerative Medicine Institute, Samsung Medical Center, Seoul, South Korea; dDepartment of Biopharmaceutical Convergence, Sungkyunkwan University, Suwon, South Korea

\section{Keywords}

Next-generation sequencing · $\mathrm{ABO}$ subgroup - Chimerism · Rare blood group - Anti-CD47 interference

\begin{abstract}
Background: Next-generation sequencing (NGS) technology has been recently introduced into blood group genotyping; however, there are few studies using NGS-based blood group genotyping in real-world clinical settings. In this study, we applied NGS-based blood group genotyping into various immunohaematology cases encountered in routine clinical practice. Methods: This study included $4 \mathrm{im}$ munohaematology cases: $A B O$ subgroup, $A B O$ chimerism, antibody to a high-frequency antigen (HFA), and anti-CD47 interference. We designed a hybridization capture-based NGS panel targeting 39 blood group-related genes and applied it to the 4 cases. Results: NGS analysis revealed a novel intronic variant (NM_020469.3:c.29-10T>G) in a patient with an $A_{e l}$ phenotype and detected a small fraction of $A B O * A 1.02$ (approximately 3-6\%) coexisting with the major genotype $A B O^{*} B .01 / 0.01 .02$ in dizygotic twins. In addition, NGS analysis found a homozygous stop-gain variant (NM_004827.3:c.376C>T, p.Gln126*;ABCG2*01N.01) in a patient with an antibody to an HFA; consequently, this patient's phenotype was predicted as Jr(a-). Lastly, blood group phenotypes predicted by NGS were concordant with
\end{abstract}

karger@karger.com www.karger.com/tmh

Karger $\frac{1}{\%}$ BOPEN ACCESS
(C) 2021 The Author(s)

Published by S. Karger AG, Basel

This is an Open Access article licensed under the Creative Commons Attribution-NonCommercial-4.0 International License (CC BY-NC) (http://www.karger.com/Services/OpenAccessLicense), applicable to the online version of the article only. Usage and distribution for commercial purposes requires written permission. those determined by serology in 2 patients treated with anti-CD47 drugs. Conclusion: NGS-based blood group genotyping can be used for identifying $A B O$ subgroup alleles, low levels of blood group chimerism, and antibodies to HFAs. Furthermore, it can be applied to extended blood group antigen matching for patients treated with anti-CD47 drugs.

(C) 2021 The Author(s)

Published by S. Karger AG, Basel

\section{Introduction}

For over a century, serological testing based on haemagglutination has been the gold standard for determining blood group phenotypes. However, there are immunohaematology problems that serological testing alone may not be able to resolve, such as ABO discrepancies. With advances in molecular methods and growing knowledge on the molecular basis of blood group antigens, blood group genotyping has emerged as a powerful tool to address such immunohaematology problems. The majority of blood group antigens result from single nucleotide variants (SNVs), rendering genotyping assay design and data interpretation straightforward [1]. This has resulted in the widespread application of SNV-based mo-

T.Y.K. and H.Y. contributed equally to this work. 
lecular methods, including PCR-restriction fragment length polymorphism, PCR sequence-specific primers, high-resolution melting analysis, matrix-assisted laser desorption/ionization time-of-flight mass spectrometry (MALDI-TOF MS), and DNA microarray, in blood group genotyping [2-5]. In particular, MALDI-TOF MS and DNA microarray, with a high-throughput capacity, provide a robust platform for large-scale blood group genotyping [2-5]. However, an inherent limitation of SNVbased molecular methods is that they can only test a limited number of SNVs; thus, comprehensive analysis of all blood group genes is not feasible [1]. To overcome this limitation, next-generation sequencing (NGS) technology has been recently introduced into blood group genotyping.

NGS-based blood group genotyping can be applied to various immunohaematology problems encountered in clinical practice. First, NGS-based blood group genotyping can be an efficient tool for identifying $A B O$ subgroup alleles, as the complete $A B O$ gene can be covered in a single assay. NGS-based blood group genotyping can also be used to detect blood group chimerism. The sensitivity of NGS for detecting chimerism is equivalent to that of realtime PCR and digital PCR (0.01-1\%) and is superior to that of fragment analysis (>1\%) [6]. Furthermore, NGSbased blood group genotyping can be used to predict rare blood group phenotypes in patients with antibodies to HFAs. SNV-based assays typically target only a handful of null alleles [7-11] whereas NGS-based assays have the potential to identify all null alleles, allowing accurate prediction of rare blood group phenotypes [12]. Lastly, NGSbased blood group genotyping can be utilized to resolve anti-CD47 interference in pretransfusion compatibility testing. Compared to SNV-based assays, NGS-based assays enable the provision of more extensively matched $\mathrm{RBC}$ units for patients treated with anti-CD47 drugs, which could reduce the risk of alloimmunization and haemolytic transfusion reactions.

Over the past several years, many groups have designed NGS-based blood group genotyping assays and addressed the technical issues encountered while validating these assays [13-22]. However, there are few studies using NGS-based blood group genotyping in real-world clinical settings. In this study, we applied NGS-based blood group genotyping into various immunohaematology cases encountered in routine clinical practice.

\section{Materials and Methods}

\section{Study Subjects and DNA Extraction}

This study included 4 immunohaematology cases at the blood bank of Samsung Medical Center, South Korea, between December 2018 and April 2020. These 4 cases were as follows: (1) ABO subgroup, (2) ABO chimerism, (3) antibody to a high-frequency antigen (HFA), and (4) anti-CD47 interference. In total, 8 Korean subjects participated in this study (ABO subgroup, 1 patient; $A B O$ chimerism, dizygotic twins and their parents; antibody to an HFA, 1 pregnant woman; anti-CD47 interference, 2 patients). Genomic DNA was isolated from whole blood using a solution-based DNA extraction kit (Wizard Genomic DNA Purification Kit; Promega, Madison, WI, USA).

\section{Serological Testing}

$\mathrm{ABO}$ forward typing was performed using the tube method with anti-A, anti-B (Shinyang Diagnostics, Siheung, South Korea), anti-A,B (Ortho Clinical Diagnostics, Raritan, NJ, USA), and anti$\mathrm{H}$ (Lorne Laboratories, Reading, UK) reagents. ABO reverse typing was conducted using the tube method with $\mathrm{A}_{1}$ and $\mathrm{B}$ cells (Ortho Clinical Diagnostics). ABO typing was also done using the gel method (DiaClon ABO/D + Reverse Grouping; BIO-RAD, Cressier, Switzerland). Adsorption and elution tests were performed to confirm the presence of A or B antigens on RBCs. Antibody screening was carried out using the tube method with a two-cell panel (Selectogen I-II; Ortho-Clinical Diagnostics) at immediate spin, $37^{\circ} \mathrm{C}$, and albumin-indirect antiglobulin test (IAT) phases. Antibody screening was also conducted using gel IAT (ID-Card LISS/ Coombs; Bio-Rad) with a two-cell panel (ID-DiaCell I-II; BioRad). In case of a positive screening result, antibody identification was performed using gel IAT with untreated and papain-treated 11-cell panels (Bio-Rad), and direct antiglobulin test (DAT) was performed using the gel method with monospecific anti-IgG and anti-C3d (DC-Screening II; Bio-Rad). Prior to initiating antiCD47 therapy, extended blood group antigen typing was performed using the tube method with anti-C, $-\mathrm{E},-\mathrm{c},-\mathrm{e},-\mathrm{K},-\mathrm{k},-\mathrm{Jk}^{\mathrm{a}}$, $-\mathrm{Jk}^{\mathrm{b}},-\mathrm{Fy}^{\mathrm{a}},-\mathrm{Fy}^{\mathrm{b}},-\mathrm{M},-\mathrm{N},-\mathrm{S}$, and $-\mathrm{s}$ reagents (Diagast, Loos, France). Patients with anti-CD47 interference in pretransfusion compatibility testing received extended antigen-matched $\mathrm{RBC}$ units guided by pretreatment serology (matched for C, E, c, e, K, Jkª $\mathrm{Jk}^{\mathrm{b}}, \mathrm{Fy}^{\mathrm{a}}$, $\mathrm{Fy}^{\mathrm{b}}, \mathrm{S}$, and $\mathrm{s}$ antigens). The strength of agglutination was graded as $0,1+, 2+, 3+$, or $4+$.

\section{Flow Cytometry Analysis}

Samples exhibiting mixed-field agglutination in ABO forward typing were analyzed using flow cytometry (FC). After three washes with phosphate-buffered saline (PBS), approximately 500,000 RBCs were added to each well of a 96-well plate containing $50 \mu \mathrm{L}$ of PBS. The RBCs were then fixed with $0.1 \%$ glutaraldehyde for 10 min at room temperature. After fixation, the plate was centrifuged at $300 \mathrm{~g}$ for $1 \mathrm{~min}$, and the supernatant was discarded. To each well, $5 \mu \mathrm{L}$ of anti-A or anti-B reagent (murine monoclonal IgM antibodies; Shinyang Diagnostics) was added, incubated for $15 \mathrm{~min}$ at room temperature, and washed twice with PBS. Then, $2 \mu \mathrm{L}$ of phycoerythrin-conjugated anti-mouse IgM secondary antibodies (BD Biosciences, San Jose, CA, USA) were added, incubated for $15 \mathrm{~min}$ at room temperature, and washed twice with PBS. Finally, the RBCs were resuspended in $200 \mu \mathrm{L}$ of PBS and analyzed on a flow cytometer (BD FACSVerse; BD Biosciences).

\section{Sanger Sequencing of ABO Exons 6 and 7}

Samples showing an $\mathrm{ABO}$ discrepancy in routine serology were subjected to Sanger sequencing of $A B O$ exons 6 and 7 as described in our previous study [23]. Briefly, PCR amplification was carried out using the forward primer ABOe6F (5'-GCTGAGTGGAGTTTCCAGGT- $\left.3^{\prime}\right)$ and the reverse primer ABOe7R ( $5^{\prime}$-AACAGGACGGACAAAGGAAA- $\left.3^{\prime}\right)$. The amplified products $(2,080 \mathrm{bp})$ were sequenced using the internal primers ABOe6R (5'-CCACCCCACTCTGTCTTGAA-3'), ABOe7F (5'-TCTGCTGCTCTAAGCCTTCC- $3^{\prime}$ ), ABOe7SF1 (5'-TCCTCAGCGAGGTGGATTAC- $\left.3^{\prime}\right)$, and ABOe7SF2 ( $5^{\prime}$-ACGAAGAGAGCCACCTGAA- $\left.3^{\prime}\right)$. 
Table 1. Results of serological testing and molecular testing (including NGS)

\begin{tabular}{|c|c|c|c|c|c|c|c|c|c|c|}
\hline \multicolumn{4}{|c|}{ Forward typing } & \multicolumn{3}{|c|}{ Reverse typing } & \multicolumn{2}{|c|}{ Adsorption and elution } & \multicolumn{2}{|l|}{ Genotype } \\
\hline Anti-A & Anti-B & Anti-A,B & Anti-H & $\mathrm{A}_{1}$ cell & B Cell & O Cell & $A_{1}$ cell & B Cell & allele 1 & allele 2 \\
\hline 0 & 0 & 0 & $4+$ & 0 & $4+$ & 0 & $2+$ & 0 & $A B O^{*} 0.01 .01$ & $A B O^{*} A 1.02$ with c. $29-10 \mathrm{~T}>\mathrm{G}$ \\
\hline
\end{tabular}

Confirmation of a Novel ABO Allele

When a novel intronic variant (NM_020469.3:c.29-10T>G) was found in a patient with a weak A phenotype, allele-specific PCR based on the c.99-288C $>$ T polymorphism (rs687621; C: A allele, T: $O$ allele) was performed using the primer pair described by Matzhold et al. [24]. Briefly, PCR amplification was conducted using the forward primerABO_In1_12,797_Amp_F(5'-GATCTGGACTGGGTTTGGAG- $3^{\prime}$ ) and the reverse primer ABO_ In2_437C/T_R ( $5^{\prime}$ - CGCCACCAGTGCCTTGG/A-3'). To determine whether the c.29-10T $>\mathrm{G}$ variant was located on the $A$ allele, the PCR products ( $655 \mathrm{bp}$ ) were sequenced using the same primer pair. Furthermore, three major regulatory regions of the $A B O$ gene, the proximal promoter, the CCAAT-binding factor NF-Y $(\mathrm{CBF} / \mathrm{NF}-\mathrm{Y})$ enhancer region, and the $+5.8-\mathrm{kb}$ site, were sequenced to exclude the possibility that a variant other than c.29$10 \mathrm{~T}>\mathrm{G}$ is responsible for the patient's weak A phenotype. The proximal promoter of the $A B O$ gene was amplified and sequenced using the primerpairmo-12F ( $5^{\prime}$-GGCGCCGTCCCTTCCTAG- $\left.3^{\prime}\right)$ and mo-12R ( $5^{\prime}$-CCTGCGGTAGCGGCTCCCT- $\left.3^{\prime}\right)$ as described previously [25]. The CBF/NF-Y enhancer region of the $A B O$ gene was amplified and sequenced using the forward primer $\left(5^{\prime}\right.$-GGAAACAAATCCTACCCCTAC- $\left.3^{\prime}\right)$ and the reverse primer ( $5^{\prime}$-GTGCTGCCTGTGCCTGTTAC- $\left.3^{\prime}\right)$ as described previously [26]. To identify two types of deletions involving the +5.8 - $\mathrm{kb}$ site of $A B O$ intron 1, a 5.8-kb deletion (c.28 +5110_10889del) and a 3.0-kb deletion (c.28+4077_7107del), PCR amplification was performed using the following primer pairs described by Sano et al. [27]: ABO+4419S (5'-TGGAATTGCTGTCTCCTCTTTTAGTCC- $3^{\prime}$ ) and ABO+11078AS (5'-GGTCCCTCCTGACCCTGACAA- $\left.3^{\prime}\right)$ for the 5.8 -kbdeletion; $\mathrm{ABO}+3043 \mathrm{~S}\left(5^{\prime}\right.$-GGAACTCCTCTGCAGTCATTCCC- $3^{\prime}$ ) and ABO+11078AS for the 3.0-kb deletion. To identify sequence variants in the $+5.8-\mathrm{kb}$ site, the PCR products were sequenced using the primer $\mathrm{ABO}+5743 \mathrm{~S}\left(5^{\prime}\right.$-GACAGTTATTCAGTTAAGACATG- ${ }^{\prime}$ ) described by Takahashi et al. [28].

\section{NGS-Based Blood Group Genotyping}

A blood group gene panel was designed to include 39 blood group-related genes ( 37 genes belonging to 34 blood group systems and 2 genes encoding an erythroid-specific transcription factor; online suppl. Table S1; for all online suppl. material, see www. karger.com/doi/10.1159/000517565). Target enrichment was performed using custom-designed capture probes (Celemics, Seoul, South Korea). The captured libraries were sequenced on an MiSeq platform (Illumina, San Diego, CA, USA) with a $2 \times 150$-bp pairedend module. Sequence reads were aligned to the hg19 reference genome using BWA-MEM (version 0.7.10), and duplicated reads were removed using Picard (version 1.138). The Genome Analysis Toolkit (GATK, version 3.5) was used for local realignment and base quality score recalibration. Variants were called using the GATK HaplotypeCaller, followed by hard filtering using the following criteria: SNVs: QualByDepth (QD) $<2.0$, FisherStrand (FS) $>60.0$, RMSMappingQuality (MQ) <40.0, MappingQualityRankSumTest (MQRankSum) $<-12.5$, or ReadPosRankSumTest (ReadPosRankSum) $<-8.0$; insertions and deletions: $Q D<2.0$, FS $>200.0$, or ReadPosRankSum $<-20.0$. Variants that passed the hard filtering were further filtered using the following criteria: depth of coverage $<10 \times$ or variant allele fraction $(\mathrm{VAF})<20 \%$ (exceptionally, in case of chimerism, the VAF threshold was set at $1 \%$ ). Variants passing through the filtering step were annotated using Variant Effect Predictor (version 94) and dbNSFP (version 4.0). After the annotated variant call format files were examined carefully, the blood group alleles of each individual were determined based on the International Society of Blood Transfusion allele database. Mapped reads were visualized using Integrative Genomics Viewer (IGV).

\section{Results}

\section{Statistics of NGS Data}

An average of 1.2 million reads were generated per sample, $42.0 \%$ of which were uniquely mapped to the target region $(70 \mathrm{~kb})$, resulting in a mean depth of coverage of $671 \times$. On average, $96.1,95.2$, and $94.1 \%$ of the target region were covered at $\geq 5 \times, 30 \times$, and $100 \times$, respectively. The coverage information for each target gene is shown in online supplementary Table S1. In all but three genes ( $R H D, R H C E$, and CR1), more than $97 \%$ of the target bases were covered at $\geq 30 \times$. The poorly covered regions of $R H D, R H C E$, and $C R 1(<30 \times)$ were as follows: $R H D$, exon 8; RHCE, exon 2 (in all samples with a CC phenotype); CR1, exons 7-13 and exons 18 and 19.

\section{Case 1: $A B O$ Subgroup}

A blood sample from a 59-year-old male patient (patient 1) was tested for the ABO type. The patient had no recent history of blood transfusion and no history of malignancy (including lymphoma and leukaemia). Table 1 summarizes the results of serological and molecular testing, including NGS. The patient's RBCs showed no agglutination with anti-A, anti- $B$, and anti-A,B reagents; however, the eluate displayed $2+$ agglutination with $A_{1}$ cells in the adsorption and elution tests. Based on these serological results, the patient was considered to have an $\mathrm{A}_{\text {el }}$ phenotype. Sanger sequencing of $A B O$ exons 6 and 7 identified two heterozygous variants (NM_020469.3:c.261delG and $c .467 \mathrm{C}>\mathrm{T}$ ); according to the sequencing results, the patient was presumed to carry the $A B O^{*} A 1.02 / O .01 .01$ genotype. Using our NGS assay covering all exons and flanking intronic regions of the $A B O$ gene, we additionally identified a novel heterozygous variant in $A B O$ intron 


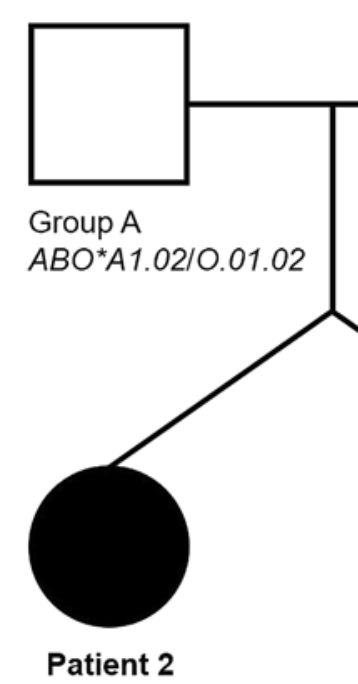

Patient 2

Serology: MF agglutination with anti-A and anti-B (stronger agglutination with anti-B than anti-A) FC analysis: group B RBCs (major population) group A RBCs (minor population) NGS analysis: $A B O^{*} B .01$ and 0.01 .02 (major alleles) $A B O{ }^{*} A 1.02$ (minor allele)

Fig. 1. Pedigree of a family with dizygotic twins showing ABO chimerism (patients 2 and 3 ).

1 (c.29-10T $>$ G). Allele-specific PCR and Sanger sequencing of the PCR products confirmed that this intronic variant was located on the $A B O^{*} A 1.02$ allele. No variant was detected in the proximal promoter, $\mathrm{CBF} / \mathrm{NF}-\mathrm{Y}$ enhancer region, and $+5.8-\mathrm{kb}$ site of the $A B O$ gene. The c.29-10T $>\mathrm{G}$ variant is absent in public databases, including dbSNP, 1000 Genomes, and gnomAD, and has not been reported in the literature previously.

\section{Case 2: $A B O$ Chimerism}

Blood samples from 10-month-old dizygotic twins (patients 2 and 3) and their parents were tested for the $\mathrm{ABO}$ type. The patients were born from a monochorionic diamniotic twin pregnancy and had no recent history of blood transfusion. The results of serological testing, FC analysis, and NGS analysis are summarized in the pedigree (Fig. 1). In the gel and tube methods, the patients' RBCs exhibited mixed-field agglutination with anti- $A$ and anti-B reagents. In the gel method, agglutination with anti-B was stronger than that with anti-A (online suppl. Fig. S1). In the gel and tube methods, the patient plasma showed no agglutination with $\mathrm{A}_{1}$ and $\mathrm{B}$ cells. FC analysis revealed two distinct $\mathrm{RBC}$ populations, group $\mathrm{B}$ RBCs (main population) and group A RBCs (minor population; online suppl. Fig. S1). The patients' father and mother were group A and group AB, respectively. Sanger sequencing of $A B O$ exons 6 and 7 was performed for the patients and their parents, and the resulting genotypes were as follows: patients 2 and 3: $A B O^{*} B .01 / O .01 .02$; father: $A B O^{*} A 1.02 / O .01 .02$; mother: $A B O^{*} A 1.02 / B .01$. Despite a thorough review of the chromatograms obtained from Sanger sequencing, we failed to detect any peaks corresponding to c.297A and c.467C $>\mathrm{T}$ (present on $A B O^{*} A 1.02$ but absent on $A B O^{*} B .01$ and $A B O^{*} 0.01 .02$ ) in the twins. However, the two variants were successfully detected using our NGS assay (VAF: c.297A, 5.8\% for patient 2 and $3.8 \%$ for patient $3 ; \mathrm{c} .467 \mathrm{C}>\mathrm{T}, 4.8 \%$ for patient 2 , and $3.2 \%$ for patient 3 ) and were confirmed by visual inspection using IGV (Fig. 2). In addition, manual phasing in IGV revealed that c.297A was in cis with c.261 G belonging to $A B O^{*} A 1.02$ (Fig. 2). Taken together, NGS analysis identified a small fraction of $A B O^{*} A 1.02$ coexisting with $A B O^{*} B .01$ and 0.01 .02 .

\section{Case 3: Antibody to an HFA}

A blood sample was collected from a pregnant woman at 29 weeks of gestation (patient 4) prior to an emergency caesarean section and submitted to our blood bank for type and screen. The patient had no previous history of blood transfusion. The patient's $\mathrm{ABO}$ and $\mathrm{RhD}$ types were group A, D-positive, and her plasma reacted with all reagent RBCs in tube IAT (1+) and gel IAT (2+). Her plasma also reacted with all the reagent $\mathrm{RBCs}$ treated with papain (3+). DAT was negative for IgG and C3d. NGS analysis identified a homozygous stop-gain variant in $A B C G 2$ exon 4 (NM_004827.3:c.376C>T, p.Gln126*; rs72552713; Fig. 3). As this variant was detected in the homozygous form, the patient's genotype was determined to be ABCG2*01N.01/01N.01. Anti-Jra human serum (from 1 group $\mathrm{AB}$ donor) and $\mathrm{Jr}(\mathrm{a}-)$ RBCs (from 1 group $\mathrm{O}$ donor) from the SCARF exchange program were used for confirmatory testing. The patient's RBCs showed no agglutination with human anti-Jra , and the patient's plasma exhibited no agglutination with $\mathrm{Jr}(\mathrm{a}-)$ RBCs. Consequently, the patient's phenotype and antibody specificity were confirmed as $\operatorname{Jr}(\mathrm{a}-)$ and anti-Jra $\mathrm{r}^{\mathrm{a}}$, respectively.

\section{Case 4: Anti-CD47 Interference}

Our blood bank was informed that 2 patients with diffuse large B-cell lymphoma (patients 5 and 6) were scheduled to begin taking ALX148, a novel anti-CD47 drug. These 2 patients enrolled in the phase 1 clinical trial of ALX148 (AT148001) were assigned to receive ALX148 at a dose of $10 \mathrm{mg} / \mathrm{kg}$ weekly. None of the patients had a recent history of blood transfusion. Prior to commencing ALX148 therapy, the patients' type and screen results were group A, D-positive with a negative antibody screen. The patients' extended blood group phenotypes determined by serology were as follows: $\mathrm{M}+\mathrm{N}+\mathrm{S}-\mathrm{s}+$; $\mathrm{C}+\mathrm{c}-\mathrm{E}-$ 
Fig. 2. Identification of a small fraction of $A B O^{*} A 1.02$ in dizygotic twins (patient 2, A; patient 3 , B) with the major genotype $A B O^{*} B .01 / O .01 .02$. The black arrows indicate three variants (c.261 G, c.297A, and c. $467 \mathrm{C}>\mathrm{T}$ ) located on $A B O^{*} A 1.02$. The VAFs of c. $297 \mathrm{~A}, c .467 \mathrm{C}>\mathrm{T}$ unique to $A B O^{*} A 1.02$ were as follows: c.297A: $5.8 \%$ for patient 2 (47 of 809 reads) and $3.8 \%$ for patient 3 ( 35 of 918 reads); c. $467 \mathrm{C}>\mathrm{T}: 4.8 \%$ for patient 2 (34 of 709 reads) and 3.2\% for patient 3 (27 of 851 reads). The red boxes indicate that c.297A is in cis with c.261 G. ${ }^{*}$ c.261 $\mathrm{G}$ has no genomic coordinate on hg19, as the $O$ allele containing c.261delG is used as a reference on hg19. Therefore, the $A$ or $B$ allele containing c.261 G appears to have an insertion(chr9:136132908_136132909insC), whereas the $O$ allele containing c.261delG appears to have no variant.
(A)

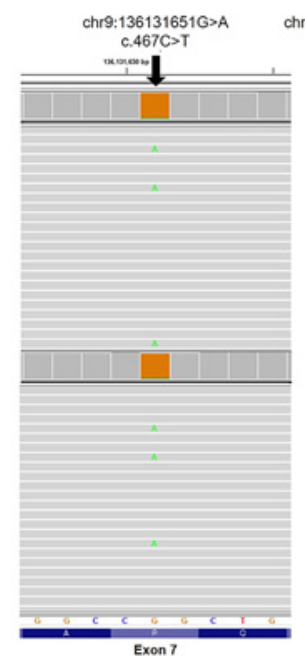

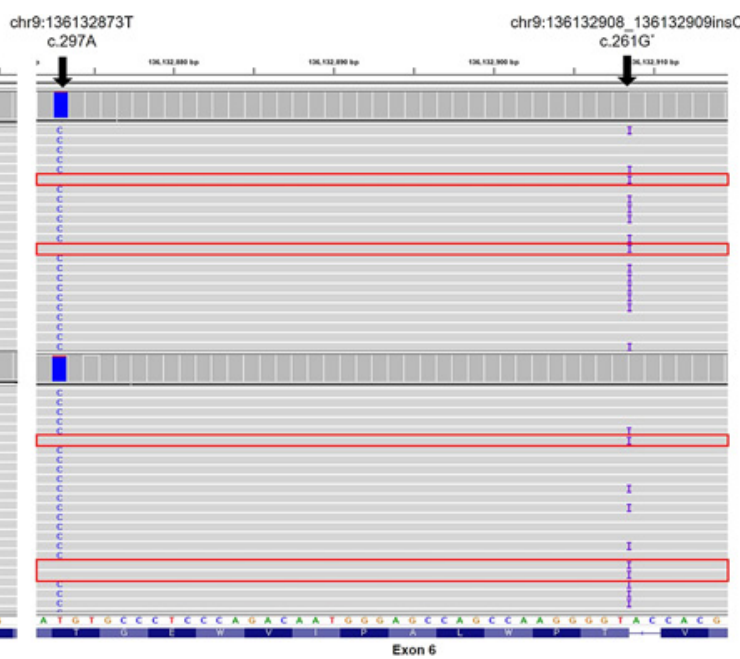

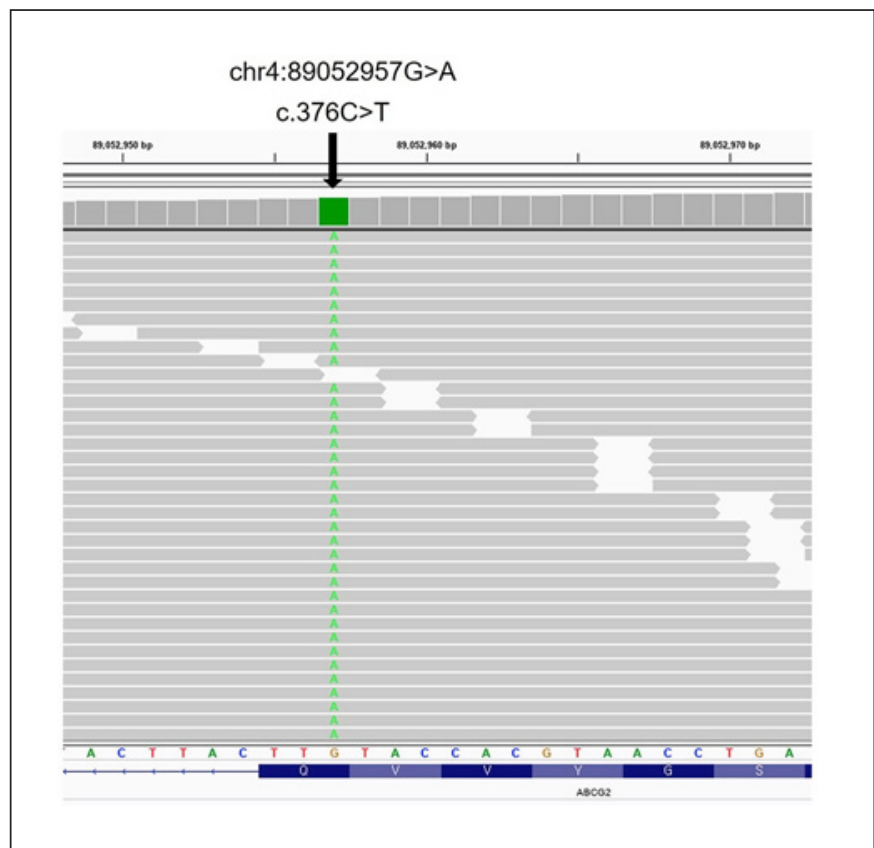

Fig. 3 Identification of a rare null allele $(A B C G 2 * 01 N .01)$ in a patient with an alloantibody to an HFA. The black arrow indicates a homozygous stop-gain variant in ABCG2 exon 4 (c.376C>T, p. Gln126*).

$\mathrm{e}+$; $\mathrm{K}-\mathrm{k}+$; Fy $(\mathrm{a}+\mathrm{b}-) ; \mathrm{Jk}(\mathrm{a}+\mathrm{b}+)$ (the same for both patients). In the initial analysis, the serologically determined blood group phenotypes were concordant with the blood group phenotypes predicted from NGS data, except for the MN phenotype (serology: $\mathrm{M}+\mathrm{N}+$; NGS: $\mathrm{M}-\mathrm{N}+$ ) (Table 2). In further analysis using IGV, a few reads containing all three variants on GYPA*01 (NM_002099.8:c.59C, c.71 G, and c.72T) were identified (Fig. 4). Accordingly, the patients' $\mathrm{MN}$ phenotypes were correctly predicted as $\mathrm{M}+\mathrm{N}+$ using NGS (Table 2). After an initial ALX148 in-

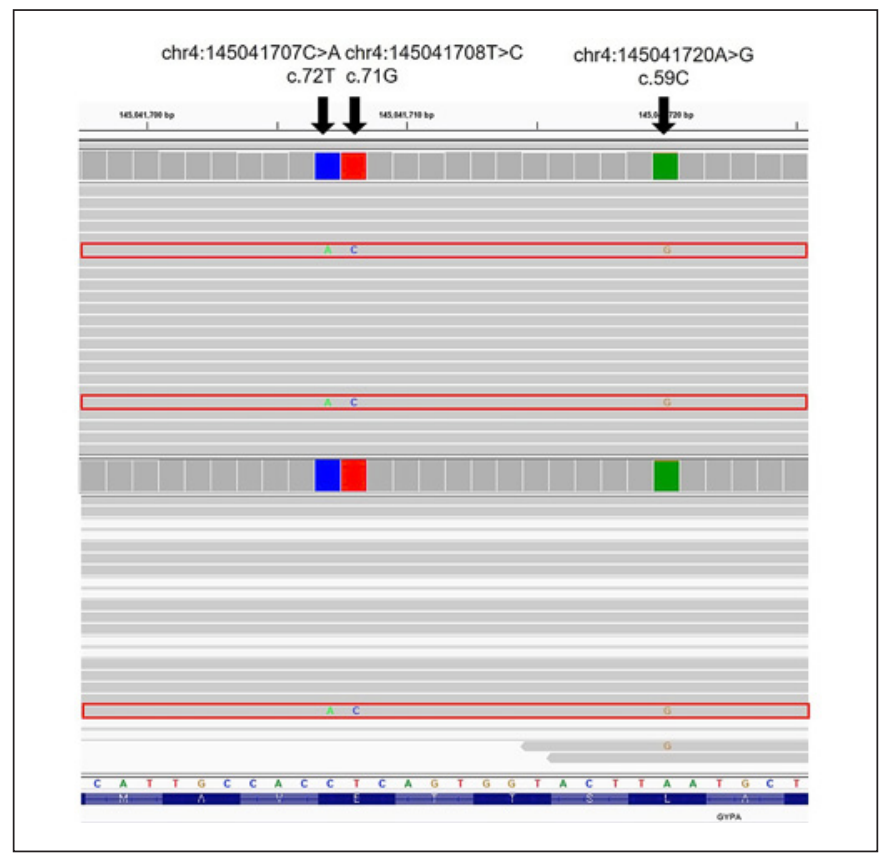

Fig. 4. Identification of $G Y P A^{*} 01$ by visual inspection using IGV. The red boxes indicate the reads containing all three variants on GYPA*01 (c.59C, c.71G, and c.72T in GYPA exon 2; black arrows). The number of reads containing all these variants were as follows: patient 5: 5 out of a total of 108 reads; patient $6: 1$ out of a total of 97 reads.

fusion, the patients' plasma exhibited panreactivity in both gel IAT (3+) and tube IAT (2+). DAT was positive for IgG (4+), but negative for C3d. During the course of ALX148 therapy, each patient received 2 units of extended antigen-matched RBCs (negative for $\mathrm{E}, \mathrm{c}, \mathrm{K}, \mathrm{Fy}^{\mathrm{b}}$, and $S$ ). Haemolytic transfusion reaction was not reported in any of the patients. 
Table 2. Comparison of blood group phenotypes predicted from NGS data and those determined by serology in 2 patients (patients 5 and 6 )

\begin{tabular}{llll}
\hline Blood group (gene) & $\mathrm{NGS}$ & & Serology \\
\cline { 2 - 3 } & genotype & phenotype & phenotype \\
\hline MNS $(G Y P A)$ & $G Y P A^{*} 02 / 02 \rightarrow G Y P A^{*} 01 / 02^{*}$ & $\mathrm{M}-\mathrm{N}+\rightarrow \mathrm{M}+\mathrm{N}+^{\mathrm{a}}$ & $\mathrm{M}+\mathrm{N}+$ \\
MNS $(G Y P B)$ & $G Y P B^{*} 04 / 04$ & $\mathrm{~S}-\mathrm{s}+$ & $\mathrm{S}-\mathrm{s}+$ \\
Rh $(R H C E)$ & $R H C E^{*} 02 / 02^{\mathrm{b}}$ & $\mathrm{C}+\mathrm{c}-\mathrm{E}-\mathrm{e}+$ & $\mathrm{C}+\mathrm{c}-\mathrm{E}-\mathrm{e}+$ \\
Kell $($ KEL) & $K E L^{*} 02 / 02$ & $\mathrm{~K}-\mathrm{k}+$ & $\mathrm{K}-\mathrm{k}+$ \\
Duffy $(A C K R 1)$ & $F Y^{*} 01 / 01$ & $\mathrm{Fy}(\mathrm{a}+\mathrm{b}-)$ & $\mathrm{Fy}(\mathrm{a}+\mathrm{b}-)$ \\
Kidd $(S L C 14 A 1)$ & $J K^{*} 01 / 02$ & $\mathrm{Jk}(\mathrm{a}+\mathrm{b}+)$ & $\mathrm{Jk}(\mathrm{a}+\mathrm{b}+)$ \\
\hline
\end{tabular}

\footnotetext{
${ }^{a}$ In both patients, all three variants on the $G Y P A^{*} 01$ allele (c.59C, c.71G, and c.72T in GYPA, exon 2) were not called by our pipeline. However, visual inspection using IGV identified a few reads containing all these variants; therefore, we corrected the patients' genotypes from GYPA*02/02 to GYPA*01/02, which was correlated with their phenotypes determined by serology $(\mathrm{M}+\mathrm{N}+) .{ }^{\mathrm{b}}$ Both patients were presumed to carry the $R H C E^{*} 02 / 02$ $\left(R H C E^{*} \mathrm{Ce} / \mathrm{Ce}\right.$ ) genotype as two of seven variants on the $R H C E^{*} 02$ allele (NM_020485.6:c.48G>C in RHCE, exon 1 and c.676G in RHCE, exon 5) were identified in the homozygous form. However, detection of the remaining five variants on the $R H C E^{*} 02$ allele (NM_020485.6:c.150C>T, c.178C>A, c.201A>G, c.203A>G, and c.307C > T in RHCE, exon 2) was not possible, as no reads were mapped to RHCE, exon 2.
}

\section{Discussion}

In all samples included in this study, most of the target region was covered at a high depth, allowing reliable detection of sequence variants in the region. On the contrary, a significant proportion of the target bases in three genes (RHD, RHCE, and CR1) were rarely covered; thus, we could not rule out the possibility that sequence variants may be present in the underrepresented region. The RHD and RHCE genes have high nucleotide sequence homology (approx. 92\%) [29]. In particular, the nucleotide sequence of $R H C E$ exon 2 in the $R H C E^{*} C$ allele is identical to that of $R H D$ exon 2, and the nucleotide sequences of exon 8 in $R H D$ and RHCE are identical [15]. Because of this sequence homology, RHCE exon 2 (in all samples with a CC phenotype) and RHD exon 8 (in all samples regardless of their RhCE phenotype) were rarely covered, which is similar to the findings of previous studies $[15,16$, 21]. The $C R 1$ gene encodes a protein carrying 30 short consensus repeats, 28 of which are arranged in four long homologous repeats [30]. Because of these repetitive sequences, a part of the $C R 1$ gene was poorly covered by our NGS assay; however, the CR1 exons 26 and 29 where all Knops blood group polymorphisms are located were adequately covered (mean depth of coverage: $252 \times$ for exon 26 and $425 \times$ for exon 29), allowing for the reliable prediction of Knops blood group phenotypes. An inherent limitation of short-read sequencing is that false-positive or false-negative variant calls can arise from the misalignment of reads to homologous regions in the genome [31]. Such a limitation can be overcome by short-read sequencing based on PCR enrichment, discriminating between homologous regions or long-read sequencing such as nanopore sequencing. A short-read sequencing method based on long-range PCR enrichment, specifically amplifying the $R H D$ gene, has already been described $[18,32]$.

The vast majority of $A B O$ subgroup-causing variants reported to date are located within $A B O$ exons 6 and 7. Therefore, when $\mathrm{ABO}$ subgroups are suspected in routine serology, the initial molecular target is limited to $A B O$ exons 6 and 7 in many blood banks, including our own. However, as $\mathrm{ABO}$ subgroup-causing variants can occur in regions other than $A B O$ exons 6 and 7 (including exons 1-5 and regulatory regions of the $A B O$ gene), though rarely [25-28, 33-35], it is necessary to develop molecular assays to screen all these regions. Wu et al.[19]recently designed a hybridization capture-based NGS assay covering the entire $A B O$ gene and identified various $A B O$ subgroup alleles, including a $5.9-\mathrm{kb}$ deletion in $A B O$ intron 1. Similarly, our study attempted to identify an $A B O$ subgroup allele by employing a hybridization capture-based NGS assay targeting 39 blood group-related genes including $A B O$; as a result, a novel intronic variant (c.29-10T $>\mathrm{G}$ in $A B O$ intron 1) was detected in a patient with an $\mathrm{A}_{\mathrm{el}}$ phenotype. The present study demonstrated that a hybridization capture-based NGS assay is useful for identifying $A B O$ subgroup alleles; however, unlike the NGS assay designed by Wu et al.[19], our NGS panel did not include important regulatory regions of the $A B O$ gene, such as the $+5.8-\mathrm{kb}$ site, and thus required additional Sanger sequencing to cover these regions. The coverage of the regulatory regions of the $A B O$ gene is particularly important in East Asians, in whom variants in these regions explain a significant proportion of $\mathrm{ABO}$ subgroups $[34,35]$. Therefore, in future studies, we will add the regulatory regions to our NGS panel. 
Intronic variants outside the canonical splice sites may cause aberrant splicing, but the effect of these variants on the splicing process should be verified through functional studies such as minigene splicing assays [36]. Although the c.29-10T $>\mathrm{G}$ variant in patient 1 is absent in population databases including gnom $A D$, it is not certain whether this variant can cause splicing defects such as exon skipping as we did not perform functional studies. In addition to functional studies, detection of transferase activity in the patient's plasma might be helpful. In this study, the presence of A antigens on the patient's RBCs was demonstrated by adsorption and elution tests. However, it is important to understand that adsorption and elution tests are prone to false-positive results [37], and therefore further FC analysis may be necessary to confirm adsorption and elution test results.

This study proved the utility of the NGS approach in detecting low levels of blood group chimerism. Our NGS assay accurately detected the minor allele $A B O^{*} A 1.02 \mathrm{co}-$ existing with the major genotype $A B O^{*} B .01 / 0.01 .02$ in dizygotic twins (patients 2 and 3). Considering the VAFs of c. $297 \mathrm{~A}$ and c. $467 \mathrm{C}>\mathrm{T}$ unique to $A B O^{*} A 1.02$, the percentage of $A B O^{*} A 1.02$ was estimated to be $3-6 \%$, which is below the detection limit of Sanger sequencing (15-20\%). This explains why unlike NGS, Sanger sequencing failed to detect $A B O^{*} A 1.02$ in the twins. Allele-specific PCR selectively amplifying minor alleles in chimeric samples can be utilized to overcome the low sensitivity of Sanger sequencing $[38,39]$; however, such procedures are timeconsuming and laborious, thus limiting their use in clinical practice. In addition to molecular methods, FC analysis can be used for detecting and quantifying blood group chimerism [40]. In the present study, FC analysis identified a small fraction of group A RBCs in the twins, which was correlated well with the findings of our NGS assay.

One option to determine the antibody specificity in a patient with an antibody to an HFA is to predict the patient's rare phenotype (HFA-negative phenotype or null phenotype) using molecular methods. In this NGS study, patient 4 was found to carry the ABCG2*01N.01/01N.01 genotype and was therefore predicted to have the $\operatorname{Jr}(\mathrm{a}-)$ phenotype, confirmed by the serological method. Without NGS, we would have needed to test numerous rare blood phenotypes such $\operatorname{Jr}(\mathrm{a}-), \mathrm{K}_{0}$, and Lan- using the serological method, which would have been costly and time consuming. SNV-based molecular methods can also be used to predict rare blood group phenotypes, but their use is usually limited to HFA-negative phenotypes such as $\mathrm{Lu}(\mathrm{b}-)$ and $\mathrm{Di}(\mathrm{b}-)$. As SNV-based molecular methods can only test a limited number of SNVs, their use may not be suitable for predicting null phenotypes with a high level of allelic heterogeneity, such as $\operatorname{Jr}(\mathrm{a}-)$ (e.g., more than 20 alleles in $A B C G 2$ are known to cause the $\operatorname{Jr}\left(\mathrm{a}^{-}\right)$phenotype [41]).
Anti-CD47 drugs such as ALX148 and Hu5F9-G4 interfere with pretransfusion compatibility testing, thus complicating the safe and timely provision of RBC units [42-44]. One possible option to resolve the anti-CD47 interference is to provide phenotypically or genotypically matched RBC units. However, it is important to understand that phenotype or genotype matching could miss clinically significant antibodies depending on the level of matching. Theoretically, NGS can identify all blood group alleles, enabling the maximum level of matching and thereby minimizing the risk of missing clinically significant antibodies. Recently, Fichou et al. [14] revealed that NGS can be applied to extended blood group antigen matching for patients with sickle cell disease. In this study, we aimed to investigate whether NGS could be applied to extended blood group antigen matching for patients treated with anti-CD47 drugs. Our NGS assay correctly predicted all the blood group phenotypes in the MNS, Rh, Kell, Duffy, and Kidd systems, demonstrating the utility of NGS-based blood group genotyping in mitigating the anti-CD47 interference in pretransfusion compatibility testing. Interestingly, our pipeline failed to detect the $G Y P A^{*} 01$ allele (encoding the $\mathrm{M}$ antigen); therefore, the patients' phenotypes were erroneously predicted to be $\mathrm{M}-$ $\mathrm{N}+$ (serology: $\mathrm{M}+\mathrm{N}+$ ). This genotyping error has been reported repeatedly and is presumed to occur due to the misalignment of GYPA exon 2 reads in $G Y P A^{*} 01$ (GYPA, $G Y P B$, and $G Y P E$ are highly homologous) [13, 16, 21, 22]. For accurate identification of $G Y P A^{*} 01$, Fichou et al. [14] developed a short-read sequencing method based on PCR enrichment, specifically amplifying the exons 1 and 2 of GYPA and GYPB [13]. In addition, GYPA*01 could be identified by relaxing variant filtering criteria such as the VAF threshold and minimum depth requirement $[16,22]$ or by detecting misaligned GYPA exon 2 reads [21]. The present study showed that visual inspection using IGV could be useful for detecting $G Y P A^{*} 01$. As reads carrying all three variants unique to $G Y P A^{*} 01$ (c.59C, c.71G, and c.72T in GYPA exon 2) are highly likely to originate from $G Y P A^{*} 01$, the false-positive rate of our method is presumed to be very low.

\section{Conclusion}

Our study demonstrated that NGS-based blood group genotyping is an effective tool for identifying $A B O$ subgroup alleles, low levels of blood group chimerism, and antibodies to HFAs. Furthermore, our study proved that NGS-based blood group genotyping can be used to resolve anti-CD47 interference in pretransfusion compatibility testing. Our findings thus suggest that NGS-based blood group genotyping can be applied to various immunohaematology cases encountered in routine clinical practice.
Kim/Yu/Phan/Jang/Cho 


\section{Statement of Ethics}

The study protocol was approved by the Institutional Review Board of Samsung Medical Center, and written informed consent was obtained from all the participants.

\section{Conflict of Interest Statement}

All authors declare that there are no conflicts of interest.

\section{Funding Sources}

There was no funding relating to this work.

\section{Author Contributions}

J.-H.J. and D.C. contributed to the conception and design of the study. H.Y. and M.-T.T.P. performed serological and molecular studies. T.Y.K. and J.-H.J. contributed to data analysis. T.Y.K., H.Y., and D.C. drafted the first version of the manuscript. All authors reviewed and edited the manuscript.

\section{References}

1 Westhoff CM. Blood group genotyping. Blood. 2019 Apr;133(17):1814-20.

2 Hillyer CD, Shaz BH, Winkler AM, Reid M. Integrating molecular technologies for red blood cell typing and compatibility testing into blood centers and transfusion services. Transfus Med Rev. 2008 Apr;22(2):117-32.

3 Anstee DJ. Red cell genotyping and the future of pretransfusion testing. Blood. 2009 Jul; 114(2):248-56.

4 Avent ND. Large-scale blood group genotyping: clinical implications. Br J Haematol. 2009 Jan;144(1):3-13.

5 McBean RS, Hyland CA, Flower RL. Approaches to determination of a full profile of blood group genotypes: single nucleotide variant mapping and massively parallel sequencing. Comput Struct Biotechnol J. 2014 Sep;11(19):147-51.

6 Andrikovics H, Örfi Z, Meggyesi N, Bors A, Varga L, Kövy P, et al. Current trends in applications of circulatory microchimerism detection in transplantation. Int J Mol Sci. 2019 Sep;20(18):4450.

7 Hashmi G, Shariff T, Zhang Y, Cristobal J, Chau C, Seul M, et al. Determination of 24 minor red blood cell antigens for more than 2000 blood donors by high-throughput DNA analysis. Transfusion. 2007 Apr;47(4):73647.

8 Polin H, Danzer M, Pröll J, Hofer K, Heilinger $\mathrm{U}$, Zopf A, et al. Introduction of a real-timebased blood-group genotyping approach. Vox Sang. 2008 Aug;95(2):125-30.

9 Jungbauer C, Hobel CM, Schwartz DW, Mayr WR. High-throughput multiplex PCR genotyping for 35 red blood cell antigens in blood donors. Vox Sang. 2012 Apr;102(3):234-42.

10 Gassner C, Meyer S, Frey BM, Vollmert C. Matrix-assisted laser desorption/ionisation, time-of-flight mass spectrometry-based blood group genotyping - the alternative approach. Transfus Med Rev. 2013 Jan;27(1): 2-9.

11 Bonet Bub C, Castilho L. ID CORE XT as a tool for molecular red blood cell typing. Expert Rev Mol Diagn. 2019 Sep;19(9):777-83.

12 Fürst D, Tsamadou C, Neuchel C, Schrezenmeier H, Mytilineos J, Weinstock C. Nextgeneration sequencing technologies in blood group typing. Transfus Med Hemother. 2020 Feb;47(1):4-13.

13 Fichou Y, Audrézet MP, Guéguen P, Le Maréchal C, Férec C. Next-generation sequencing is a credible strategy for blood group genotyping. Br J Haematol. 2014 Nov; 167(4):554-62.

14 Fichou Y, Mariez M, Le Maréchal C, Férec C. The experience of extended blood group genotyping by next-generation sequencing (NGS): investigation of patients with sicklecell disease. Vox Sang. 2016 Nov;111(4):41824.

15 Schoeman EM, Lopez GH, McGowan EC, Millard GM, O'Brien H, Roulis EV, et al. Evaluation of targeted exome sequencing for 28 protein-based blood group systems, including the homologous gene systems, for blood group genotyping. Transfusion. 2017 Apr; 57(4):1078-88.

16 Lane WJ, Westhoff CM, Gleadall NS, Aguad M, Smeland-Wagman R, Vege S, et al. Automated typing of red blood cell and platelet antigens: a whole-genome sequencing study. Lancet Haematol. 2018 Jun;5(6):e241-e51.

17 Orzinska A, Guz K, Mikula M, Kulecka M, Kluska A, Balabas A, et al. A preliminary evaluation of next-generation sequencing as a screening tool for targeted genotyping of erythrocyte and platelet antigens in blood donors. Blood Transfus. 2018 May;16(3):28592.

18 Tounsi WA, Madgett TE, Avent ND. Complete RHD next-generation sequencing: establishment of reference RHD alleles. Blood Adv. 2018 Oct;2(20):2713-23.

19 Wu PC, Lin YH, Tsai LF, Chen MH, Chen PL, Pai SC. ABO genotyping with next-generation sequencing to resolve heterogeneity in donors with serology discrepancies. Transfusion. 2018 Sep;58(9):2232-42.

20 Jakobsen MA, Dellgren C, Sheppard C, Yazer $\mathrm{M}$, Sprogøe U. The use of next-generation sequencing for the determination of rare blood group genotypes. Transfus Med. 2019 Jun; 29(3):162-8.

21 Lane WJ, Vege S, Mah HH, Lomas-Francis C, Aguad M, Smeland-Wagman R, et al. Automated typing of red blood cell and platelet antigens from whole exome sequences. Transfusion. 2019 Oct;59(10):3253-63.

22 Roulis E, Schoeman E, Hobbs M, Jones G, Burton M, Pahn G, et al. Targeted exome sequencing designed for blood group, platelet, and neutrophil antigen investigations: proofof-principle study for a customized single-test system. Transfusion. 2020 Sep;60(9):210820.

23 Cho D, Kim SH, Jeon MJ, Choi KL, Kee SJ, Shin MG, et al. The serological and genetic basis of the cis-AB blood group in Korea. Vox Sang. 2004 Jul;87(1):41-3.

24 Matzhold EM, Drexler C, Wagner A, Bernecker C, Pessentheiner A, Bogner-Strauß JG, et al. A 24-base pair deletion in the $\mathrm{ABO}$ gene causes a hereditary splice site defect: a novel mechanism underlying $\mathrm{ABO}$ blood group $\mathrm{O}$. Transfusion. 2020 Jul;60(7):1564-72.

25 Olsson ML, Irshaid NM, Hosseini-Maaf B, Hellberg A, Moulds MK, Sareneva H, et al. Genomic analysis of clinical samples with serologic ABO blood grouping discrepancies: identification of 15 novel A and B subgroup alleles. Blood. 2001 Sep;98(5):158593.

26 Seltsam A, Wagner FF, Grüger D, Gupta CD, Bade-Doeding C, Blasczyk R. Weak blood group $\mathrm{B}$ phenotypes may be caused by variations in the CCAAT-binding factor/NF-Y enhancer region of the $\mathrm{ABO}$ gene. Transfusion. 2007 Dec;47(12):2330-5.

27 Sano R, Nakajima T, Takahashi K, Kubo R, Kominato Y, Tsukada J, et al. Expression of $\mathrm{ABO}$ blood-group genes is dependent upon an erythroid cell-specific regulatory element that is deleted in persons with the Bm phenotype. Blood. 2012 May;119(22):5301-10.

28 Takahashi Y, Isa K, Sano R, Nakajima T, Kubo $\mathrm{R}$, Takahashi K, et al. Presence of nucleotide substitutions in transcriptional regulatory elements such as the erythroid cell-specific enhancer-like element and the $\mathrm{ABO}$ promoter in individuals with phenotypes A3 and B3, respectively. Vox Sang. 2014 Aug;107(2):17180.

29 Okuda H, Kajii E. The evolution and formation of RH genes. Leg Med. 2002 Sep;4(3): 139-55.

30 Sandri TL, Lidani KCF, Andrade FA, Meyer CG, Kremsner PG, de Messias-Reason IJ, et al. Human complement receptor type 1 (CR1) protein levels and genetic variants in chronic Chagas disease. Sci Rep. 2018 Jan;8(1):526.

31 Mandelker D, Schmidt RJ, Ankala A, McDonald Gibson K, Bowser M, Sharma $\mathrm{H}$, et al. Navigating highly homologous genes in a molecular diagnostic setting: a resource for clinical next-generation sequencing. Genet Med. 2016 Dec;18(12):1282-9.

32 Tammi SM, Tounsi WA, Sainio S, Kiernan M, Avent ND, Madgett TE, et al. Next-generation sequencing of 35 RHD variants in 16253 serologically D- pregnant women in the Finnish population. Blood Adv. 2020 Oct;4(20): 4994-5001. 
33 Seltsam A, Blasczyk R. Missense mutations outside the catalytic domain of the ABO glycosyltransferase can cause weak blood group A and B phenotypes. Transfusion. 2005 Oct 45(10):1663-9.

34 Ying Y, Hong X, Xu X, Ma K, He J, Zhu F. A novel mutation $+5904 \mathrm{C}>\mathrm{T}$ of RUNX1 site in the erythroid cell-specific regulatory element decreases the $\mathrm{ABO}$ antigen expression in Chinese population. Vox Sang. 2018 Aug;113(6): 594-600.

35 Ogasawara K, Miyazaki T, Ito S, Yabe R, Uchikawa M, Enomoto T, et al. The B allele with a $5.8 \mathrm{~kb}$ deletion in intron 1 of the $\mathrm{ABO}$ gene is the major allele in Japanese individuals with $\mathrm{Bm}$ and $\mathrm{A} 1 \mathrm{Bm}$ phenotypes. Vox Sang. 2018 May;113(4):393-6.

36 Anna A, Monika G. Splicing mutations in human genetic disorders: examples, detection, and confirmation. J Appl Genet. 2018 Aug; 59(3):253-68
37 Kim TY, Hong YJ, Kim MJ, Kim H, Kim TS, Park JS, et al. Recommendations regarding practical DEL typing strategies for serologically D-negative Asian donors. Transfus Med Hemother. 2020 Feb;47(1):88-93.

38 Cho D, Lee SK, Yazer MH, Shin MG, Shin JH, Suh SP, et al. A dispermic chimera with mixed field blood group B and mosaic 46,XY/47,XYY karyotype. J Korean Med Sci. 2007 Jun;22(3): 553-6.

39 Won EJ, Park HR, Park TS, Oh SH, Shin MG, Shin JH, et al. Amplification refractory mutation system-PCR is essential for the detection of chimaeras with a minor allele population: a case report. J Clin Pathol. 2013 May;66(5): 446-8.

40 Hult AK, Olsson ML. Many genetically defined $\mathrm{ABO}$ subgroups exhibit characteristic flow cytometric patterns. Transfusion. 2010 Feb;50(2):308-23.
41 International Society of Blood Transfusion. Red cell immunogenetics and blood group terminology. Amsterdam: ISBT. Available from: http://www.isbtweb.org/working-parties/red-cell-immunogenetics-and-bloodgroup-terminology/(accessed April 26, 2021)

42 Brierley CK, Staves J, Roberts C, Johnson H, Vyas P, Goodnough LT, et al. The effects of monoclonal anti-CD47 on RBCs, compatibility testing, and transfusion requirements in refractory acute myeloid leukemia. Transfusion. 2019 Jul;59(7):2248-54.

43 Velliquette RW, Aeschlimann J, Kirkegaard J, Shakarian G, Lomas-Francis C, Westhoff CM. Monoclonal anti-CD47 interference in red cell and platelet testing. Transfusion. 2019 Feb;59(2):730-7.

44 Kim TY, Yoon MS, Hustinx H, Sim J, Wan HI, $\mathrm{Kim} \mathrm{H}$. Assessing and mitigating the interference of ALX148, a novel CD47 blocking agent, in pretransfusion compatibility testing. Transfusion. 2020 Oct;60(10):2399-407. 